\title{
Complete Einstein equations from the generalized First Law of Entanglement
}

\author{
Eunseok Oh, ${ }^{1, *}$ I. Y. Park, ${ }^{2, \dagger}$ and Sang-Jin Sin ${ }^{1, \$}$ \\ ${ }^{1}$ Department of Physics, Hanyang University, Seoul 04763, Korea \\ ${ }^{2}$ Department of Applied Mathematics, Philander Smith College Little Rock, Arkansas 72223, USA
}

(Received 25 September 2017; published 13 July 2018)

\begin{abstract}
Recently, it was observed that the first law of entanglement leads to the linearized Einstein equation. In this paper, we point out that the gravity dual of an relative entropy expression is equivalent to the full nonlinear Einstein equation. We also construct an entanglement vector field $V_{E}$ whose flux is the entanglement entropy. The flow of the vector field looks like sewing two space regions along the interface.
\end{abstract}

DOI: 10.1103/PhysRevD.98.026020

\section{INTRODUCTION}

One of the most inspiring ideas in the recent development of string theory is the suggestion [1,2] that the classical spacetime is a consequence of the quantum entanglement without which two nearby regions of spacetime would come apart [1,2] and, moreover, that the Einstein equation itself is coming from a relation of entanglement entropy at least at the linearized level [3]. The latter is a consequence of connecting two different descriptions of entanglement entropy (EE): one as the area of the Ryu-Takayanagi surface [4] and the other as the expectation value of the modular Hamiltonian [5]. Later, it was pointed out [6] that such relationship between the first law of $\mathrm{EE}$ and the linearized gravity equation are connected through the off-shell Noether theorem formulated by Wald [7-11].

Deriving the Einstein equation from the first lawis quite similar to the activity of the 1990s led by the work of Jacobson [12]: assuming the thermodynamic first law, he derived the gravity equation. The difference in the recent activity $[3,6]$ is that the entanglement first law and its gravity dual are derived from the conformal field theory (CFT), although it gives only a linearized equation. That is, recent activities aim to derive the Einstein equation of the dual gravity of a CFT assuming the presence of holography. In Ref. [13], the authors extended the program to the nonlinear second order in the perturbative scheme. The

\footnotetext{
1spk.1pg@gmail.com †inyongpark05@gmail.com

*sangjin.sin@gmail.com
}

Published by the American Physical Society under the terms of the Creative Commons Attribution 4.0 International license. Further distribution of this work must maintain attribution to the author(s) and the published article's title, journal citation, and DOI. Funded by SCOAP. major efforts of Ref. [13] are devoted to deriving the "gravity dual expression of the relative entropy" (GDERE) starting from CFT up to second order.

While proving the GDERE from the CFT to all orders is yet to be done, we can still ask "if we assume this part is done, does it imply the full nonlinear Einstein equation?" The goal of this paper is to prove that the answer is "yes." As we will see later, having the GDERE gives the gravitational form of the generalized first law of entanglement entropy and it is equivalent to the Einstein equation.

The other goal of this paper is to construct a vector field associated with the EE whose flux is the EE independent of the surface over which the vector field is integrated. The flux line, once the total flux is quantized, is analogous to the microscopic wormhole and is concentrated along the boundary of the entangled regions.

\section{EINSTEIN EQUATION FROM ENTANGLEMENT IN LINEAR ORDER}

To set up notation, we start with a short review of relevant concepts. Given a physical state given by a density matrix $\rho$ and a ball-like region $B$ of radius $R$, one can decompose the Hilbert space into tensor product $\mathcal{H}=$ $\mathcal{H}_{B} \otimes \mathcal{H}_{\bar{B}}$, where $\mathcal{H}_{B}$ is the Hilbert space of local fields over $B$. The reduced density operator $\rho_{B}=\operatorname{Tr}_{\mathcal{H}_{\bar{B}}} \rho$. The entanglement entropy is given by $S_{B}=-\operatorname{Tr} \rho_{B} \ln \rho_{B}$. From now on, we delete the subscript $B$ when there is no confusion. The modular Hamiltonian $H_{0}=-\log \rho_{0}$ for a reference state $\rho_{0}$ which is normalized by $\operatorname{Tr} \rho_{0}=1$. If we call the expectation value of the modular Hamiltonian for the state $\rho$ the "energy" of the state $\rho$, then we have $E=\left\langle H_{0}\right\rangle=-\operatorname{Tr} \rho \ln \rho_{0}$. Under finite variation of the state from $\rho_{0}$ to $\rho$, we have the following identity

$$
\Delta E-\Delta S=S\left(\rho \mid \rho_{0}\right),
$$




$$
\begin{gathered}
\text { where } \Delta E=-\operatorname{Tr}\left(\rho-\rho_{0}\right) \ln \rho_{0}, \\
\Delta S=-\operatorname{Tr} \rho \ln \rho+\operatorname{Tr} \rho_{0} \ln \rho_{0}, \\
\left.S\left(\rho \mid \rho_{0}\right)=\operatorname{Tr} \rho\left(\ln \rho-\ln \rho_{0}\right)\right) .
\end{gathered}
$$

Three important remarks are in order. First, (1) and (4) can be used as the definition and, as a result, interchangeably. Second, $\Delta E$ is not a total variation while $\Delta S$ is, because the relative entropy, $S\left(\rho \mid \rho_{0}\right)$, cannot be so. Similar phenomena will be observed in their gravitational versions. Finally, the relative entropy is always positive [14], and this is the origin of the entanglement first law: as a function of $\rho$, $S\left(\rho \mid \rho_{0}\right)$ is minimal at the reference state. Such an extremality condition is the usual entanglement first law,

$$
\delta E-\delta S=0,
$$

where $\delta f=\left.\frac{d}{d \lambda} f\right|_{\lambda=0}$ for $f$, which is a one-parameter family of $\lambda \in[0, \varepsilon]$. The positivity of the relative entropy is also related to the positivity of energy $[15,16]$ and that of the Fisher metric for information theory [17]. Both terms of the first law can be calculated in gravitational languages using the AdS/CFT and Ryu-Takayanagi formula, and it turns out that the first law leads to the linearized Einstein equation as we will review below.

Suppose the density operator depends on parameters $R^{1}, R^{2}, \ldots, R^{M}$ which we symbolically denote by a vector $\mathbf{R}$ and let $\rho_{0}=\rho\left(\mathbf{R}_{0}\right)$ and $\rho=\rho\left(\mathbf{R}_{1}\right)$ for some $\mathbf{R}_{0}, \mathbf{R}_{1}$. Introducing the modular potential $V=-\ln \rho$ and the modular force $F_{\alpha}=-\nabla_{\alpha} V$ in the parameter space, we can express the relative entropy as

$$
S\left(\rho \mid \rho_{0}\right)=\left\langle\int_{C} d \mathbf{R} \cdot \mathbf{F}\right\rangle,
$$

which can be interpreted as the "work", $W$, done on the system by $\mathbf{F}$ to change the system from $\rho_{0}$ to $\rho$. Notice that it is independent of the path $\mathrm{C}$ connecting $\rho_{0}$ and $\rho$ of the integration. Then the identity (1) itself, although in a finite difference form, can be considered as a first law,

$$
\Delta E-\Delta S=W=S\left(\rho \mid \rho_{0}\right),
$$

which we call the "generalized entanglement first law." In fact, it has a gravity version. Our claim is that while we get the linearized gravity equation by using (5), we will get the full nonlinear equation if we use the gravity version of (7).

For any CFT vacuum $\rho_{0}=|0\rangle\langle 0|$, a conformal mapping can be constructed which maps the causal development of the ball $B$ to a hyperbolic cylinder $H^{d-1} \times R_{\tau}$ and $\rho_{0}$ to a thermal density operator $\exp \left(-2 \pi R H_{\tau}\right)$ of CFT on hyperbolic space. Namely, the vacuum state is mapped to a thermal state of temperature $T=1 / 2 \pi R$ on the $H^{d-1}$ and the modular Hamiltonian actually generates the time evolution of CFT on the hyperbolic space. According to the AdS/CFT, the thermal state on $H^{d-1}$ can be represented by a AdS black hole with temperature $T=1 / 2 \pi R$ and the AdS-Rindler space, which can be figured as a patch of AdS space with Poincare metric.

As described above, the Hamiltonian $H_{\tau}=\int_{H^{d-1}} T_{t t}$ is equal to the unitarily transformed modular Hamiltonian of the original CFT in the flat space [5]: $H_{0}=2 \pi R U \tilde{H}_{\tau} U^{-1}$. Using this, the authors of [5] expressed the modular Hamiltonian $H_{0}$ in terms of the energy-momentum tensor of CFT,

$$
H_{0}=2 \pi \int_{B} d^{d-1} x \frac{R^{2}-|\vec{x}|^{2}}{2 R} T_{t t}=\int_{B} d \sigma^{\mu} \zeta_{B}^{\nu} T_{\mu \nu},
$$

where $\vec{x}=0$ is located at the center of the ball of radius $R$, and $\zeta_{B}^{\mu}$ is the pullback of the Killing vector $\frac{\partial}{\partial \tau}$ by the mapping that maps the causal development of $B$ to the hyperbolic cylinder $H^{d-1} \times R_{\tau}$. It can be considered as the boundary restriction of a Killing vector $\xi$ of AdS which vanishes at $\tilde{B}$. More explicitly,

$$
\xi_{B}=\frac{\pi}{R}\left[R^{2}-z^{2}-t^{2}-x^{i} x_{i}\right] \partial_{t}-\frac{2 \pi}{R} t\left[z \partial_{z}+x^{i} \partial_{i}\right],
$$

and $\zeta_{B}=\lim _{z \rightarrow 0} \xi_{B}$. The entanglement energy $E_{B}$ is given by $E_{B}=\int_{B} \zeta_{B}^{\mu}\left\langle T_{\mu \nu}\right\rangle d \sigma^{\nu}$. Now, the gravitational dual of $\delta E_{B}$ is readily given since the AdS/CFT dictionary gives the relation between the expectation value of the energy-momentum tensor and the metric variation, $\left\langle T_{\mu \nu}\right\rangle \sim z^{d-2} \delta g_{\mu \nu}$. The gravitational dual of $\delta S_{B}$ can be given using the Ryu-Takayanagi prescription $\mathcal{S}_{B}=$ Area $[\tilde{B}] / 4 G_{N}$ [4]. The crucial observation of [6] is that there exists a $d-1$ form $\chi$ in asymptotic $\operatorname{AdS}_{d+1}$ such that

$$
\int_{B} \chi=\delta E_{B}^{\text {grav }}, \text { and } \quad \int_{\tilde{B}} \chi=\delta S_{B}^{\text {grav }}
$$

based on the formalism of Iyer-Wald [8,9],

$$
\delta E_{B}^{\mathrm{grav}}-\delta S_{B}^{\mathrm{grav}}=\int_{B-\tilde{B}} \chi=\int_{\Sigma} d \chi,
$$

where $\Sigma$ is the $t=0$ slice whose boundaries are $B$ and $\tilde{B}$. Since it turns out to be

$$
d \chi=-2 \xi_{B}^{a} \delta E_{a b} \epsilon^{b},
$$

the entanglement first law implies the linearized Einstein equation $\delta E_{a b}=0$.

Since understanding Wald's formalism is essential for later formalism, we describe it below shortly. Start from the Lagrangian written in differentiable form notation: $\mathbf{L} \equiv L[\phi] \boldsymbol{\epsilon}$, where $\phi$ is a collective representation of the 
bulk fields including the metric and $\boldsymbol{\epsilon}$ is the volume form. The general variation of $\mathbf{L}$ can be written as

$$
\delta \mathbf{L}[\phi]=\mathbf{E}^{\phi} \delta \phi+d \Theta[\delta \phi],
$$

where $\mathbf{E}^{\phi}$ denotes field equations and $\Theta$, the symplectic potential current that contains a Gibbons-Hawking term. When the variation is a diffeomorphism generated by a vector field $\xi, \delta_{\xi} \mathbf{L}=d(\xi \cdot \mathbf{L})$ since $\delta_{\xi}=i_{\xi} d+d i_{\xi}$ and $\mathbf{L}$ is the top form. In terms of the Noether current codimensionone form,

$$
\mathbf{J}_{\xi}=\Theta\left[\delta_{\xi} \phi\right]-\xi \cdot \mathbf{L},
$$

Eq. (13) for the diffeomorphic variation is

$$
d \mathbf{J}_{\xi}=-\mathbf{E}^{\phi} \cdot \delta_{\xi} \phi
$$

so that $\mathbf{J}$ is the closed form for the fields at on-shell. Therefore $\mathbf{J}_{\xi}=d \mathbf{Q}_{\xi}$ at on-shell. For off-shell, one can show $[6,9]$ that

$$
\mathbf{J}_{\xi}=d \mathbf{Q}_{\xi}+\xi^{a} \mathbf{C}_{a}
$$

where $\mathbf{C}_{a}$ 's are constraints which vanish for the metric satisfying the equation of motion [8]:

$$
\begin{aligned}
& \mathbf{Q}=\frac{1}{16 \pi G_{N}} \nabla^{a} \xi^{b} \boldsymbol{\epsilon}_{a b}, \quad \mathbf{C}_{a}=2 E_{a b}^{g} \boldsymbol{\epsilon}^{b}, \\
& \text { with } \quad E_{a b}^{g}=\frac{1}{8 \pi G_{N}}\left(R_{a b}-\frac{1}{2} g_{a b} R\right)-T_{a b}^{m} .
\end{aligned}
$$

On the other hand, if we introduce $\boldsymbol{\omega}$, a two-form in phase space but codimension-one form in spacetime, by

$$
\boldsymbol{\omega}\left(\phi ; \delta_{1} \phi, \delta_{2} \phi\right)=\delta_{1} \Theta\left(\delta_{2} \phi\right)-\delta_{2} \Theta\left(\delta_{1} \phi\right),
$$

we can express $\mathbf{J}_{\xi}$ in terms of $\boldsymbol{\omega}$ as follows:

$$
\delta \mathbf{J}_{\xi}=\boldsymbol{\omega}\left(\delta \phi, \delta_{\xi} \phi\right)+d(\xi \cdot \Theta(\delta \phi))-\xi \cdot \mathbf{E}^{\phi} \delta \phi .
$$

Using Eqs. (16) and (19), we get an off-shell relation,

$$
\begin{aligned}
& d \chi=\omega\left(\delta \phi, \delta_{\xi} \phi\right)-\xi \cdot\left(\delta \mathbf{C}+\mathbf{E}^{\phi} \delta \phi\right), \\
& \text { with } \chi=\delta \mathbf{Q}_{\xi}-\xi \cdot \Theta(\delta \phi) .
\end{aligned}
$$

So far $\delta$ is an infinitesimal variation defined by $\delta \phi=\left.\frac{d}{d \lambda} \phi(x ; \lambda)\right|_{\lambda=0}$. The point of Holland and Wald [11] is that if we replace $\delta \rightarrow \frac{d}{d \lambda}$ without setting $\lambda=0$ after the derivative, all the steps above go through so that we now have an all-orders relation in $\lambda \in[0, \varepsilon]$. Then Eq. (20) can be replaced by

$$
\begin{aligned}
d \chi & =\omega\left(\frac{d}{d \lambda} \phi, \delta_{\xi} \phi\right)-\xi \cdot\left(\frac{d}{d \lambda} \mathbf{C}+\mathbf{E}^{\phi} \cdot \frac{d}{d \lambda} \phi\right), \text { with } \\
\chi & =\frac{d}{d \lambda} \mathbf{Q}_{\xi}-\xi \cdot \Theta\left(\frac{d}{d \lambda} \phi\right)
\end{aligned}
$$

An important remark is that we should work in the HollandWald gauge [11], where the Ryu-Takayanagi surface and $\xi$ do not change their coordinate dependence for any metric deformation $g(x ; \lambda)$ with $\lambda \in[0, \varepsilon]$, which gives the restriction to the size of $\varepsilon$.

Notice also that, for the linear order, the canonical energy term becomes $\boldsymbol{\omega}\left(g_{0} ; \delta g, \delta_{\xi_{B}} g_{0}\right)$, and it vanishes for the AdS metric $g_{0}$ since $\delta_{\xi_{B}} g_{0}=0$. Notice also in Eq. (12), $\xi \cdot \mathbf{E}^{g} \cdot \delta g$ does not appear either, because the explicit form of the AdS metric was already used to give $\mathbf{E}\left[g_{0}\right]=0$. However, for nonlinear order, one has to consider a finite variation $g(\varepsilon)$ and consider the cotangent space of the space of metric at $g(\lambda)$ for arbitrary $\lambda$ between 0 and $\varepsilon$. In this case, neither of the two vanish, and this fact provides the main source of the nontriviality in getting the nonlinear gravity equation.

\section{NONLINEAR EINSTEIN EQUATION FROM ENTANGLEMENT}

The issue of the full Einstein equation was discussed earlier in [18-21] and most notably in [13], where the program of getting gravity equations starting from CFT is extended perturbatively to second order. An essential part of the above paper is to derive the gravity expression of relative entropy starting from the CFT up to the second order. Similar efforts have been made in [20]. Given the fact that completing this program to all orders is certainly nontrivial, one may ask whether, if this part is assumed to be proven to all orders, we can actually show that the full nonlinear Einstein equation can be implied from there. This question can be addressed purely in a gravitational context, because as we will see shortly, the gravity expression of relative entropy can be derived from the Holland-Wald offshell identity by imposing the Einstein equation. One can ask the reverse question, namely, can we derive the Einstein equation from the relative entropy expression. We will see that the answer is positive.

To simplify the setting, we consider only pure gravity so that $\phi(x ; \lambda)$ is replaced by the metric $g(x ; \lambda)$, and we choose $\xi$ as the Killing vector of AdS given in Eq. (9).

Integrating both sides of Eq. (20) over $\Sigma$, whose boundary is $B$ and $\tilde{B}$, we get Eqs. (11) and (12). By integrating (21) over $\Sigma$, the region between $B$ and $\tilde{B}$ at the time slice $t=0$, we have $[11,16]$

$$
\begin{aligned}
& \int_{B} \boldsymbol{\chi}-\int_{\tilde{B}} \boldsymbol{\chi}=\int_{\Sigma} \omega\left(g_{\lambda} ; \frac{d}{d \lambda} g_{\lambda}, \delta_{\xi_{B}} g_{\lambda}\right)+\int_{\Sigma}(\hat{E}+\hat{C}), \\
& \text { where } \hat{E}=-\xi_{B}^{a} \boldsymbol{\epsilon}_{a} E_{b c}^{g}\left[g_{\lambda}\right] \frac{d}{d \lambda} g^{b c}, \quad \hat{C}=-\xi_{B}^{a} \frac{d}{d \lambda} \mathbf{C}_{a}\left[g_{\lambda}\right] .
\end{aligned}
$$


We first consider only physical metrics which satisfy equations of motion, then $\hat{E}=\hat{C}=0$, so that

$$
\int_{B} \chi-\int_{\tilde{B}} \chi=\int_{\Sigma} \omega\left(g_{\lambda} ; \frac{d}{d \lambda} g_{\lambda}, \delta_{\xi_{B}} g_{\lambda}\right) .
$$

Notice that the right-hand side is not zero since $\xi_{B}$ is the Killing vector of the background metric $g_{0}$, not that of $g_{\lambda}$. One should also notice that the first term of (23) is not a total variation as one can see in (21) and, therefore, cannot be written, in general, as $\frac{d}{d \lambda} E_{B}^{\text {grav }}$, while the second term is always a total variation so that it can be written as $\frac{d}{d \lambda} S_{B}^{\text {grav }}$. Integrating Eq. (23) by $\int_{0}^{\varepsilon} d \lambda$, we have

$$
\Delta E_{B}^{\mathrm{grav}}-\Delta S_{B}^{\mathrm{grav}}=\int_{0}^{\varepsilon} d \lambda \int_{\Sigma} \omega\left(g ; \frac{d}{d \lambda} g, \delta_{\xi_{B}} g\right),
$$

where

$$
\Delta E_{B}^{\mathrm{grav}}=\int_{0}^{\varepsilon} d \lambda \int_{B} \chi, \quad \Delta S_{B}^{\mathrm{grav}}=\int_{0}^{\varepsilon} d \lambda \int_{\tilde{B}} \chi .
$$

Since one can "define" the relative entropy as the difference between $\Delta E$ and $\Delta S$ as we noted earlier, Eq. (24) can be used to identify the gravity version of relative entropy [16],

$$
S^{\text {grav }}\left(\rho \mid \rho_{0}\right)=\int_{0}^{\varepsilon} d \lambda \int_{\Sigma} \omega\left(g ; \frac{d}{d \lambda} g, \delta_{\xi_{B}} g\right) .
$$

Then, Eq. (24) becomes

$$
\Delta E_{B}^{\text {grav }}-\Delta S_{B}^{\text {grav }}=S^{\text {grav }}\left(\rho \mid \rho_{0}\right),
$$

which is nothing but the gravity dual of the generalized first law (7).

So far, we have seen that the on-shell expression of the Holland-Wald identity gives the gravitational version of the generalized first law. This has been discussed in Refs. [13,16,17,22]. The authors of [13] proved the differential version of (24) from the CFT up to second order, which enabled them to prove the Einstein equation to the corresponding order.

What we want to do is the reverse direction: if a metric satisfies the gravity version of the generalized entanglement first law, it should satisfy the Einstein equation. In other words, we want to prove that the gravity expression of the relative entropy, Eq. (25), or its consequence (24), is equivalent to the equation of motion. This is not a tautology. Notice that deriving (25) from CFT is not our goal.

Namely, we want to derive the full Einstein equation, starting from Eq. (24). By integrating Eq. (22) into $\lambda$ over $[0 . \varepsilon]$, we first rewrite it as

$$
\Delta E_{B}^{\mathrm{grav}}-\Delta S_{B}^{\mathrm{grav}}-S^{\mathrm{grav}}\left(\rho \mid \rho_{0}\right)=\int_{0}^{\varepsilon} d \lambda \int_{\Sigma}(\hat{E}+\hat{C}),
$$

Now if we impose Eq. (24) or (26), which is the gravity dual of the generalized first law of entanglement, the righthand side of the above equation vanishes. Taking the derivative of the equation with respect to $\varepsilon$, we get

$$
\hat{E}[g(\varepsilon)]+\hat{C}[g(\varepsilon)]=0 .
$$

Using the explicit form of the constraint given in (17), we have

$$
\xi_{b} E^{c d}[g(\varepsilon)] g^{\prime}(\varepsilon)+2 \xi^{a} E_{a b}^{\prime}[g(\varepsilon)]=0,
$$

where the prime denotes $\frac{d}{d \varepsilon}$ and we deleted the subscript/ superscript $g, B$ from $E$ to simplify the notation. We expand the $E_{a b}[g(\varepsilon)]$ and $g_{a b}(\varepsilon)$ in $\varepsilon$ :

$$
E[g(\varepsilon)]=\sum_{n=0}^{\infty} \varepsilon^{n} E^{(n)}, \quad \text { and } \quad g(\varepsilon)=\sum_{n=0}^{\infty} \varepsilon^{n} g^{(n)} .
$$

Then, Eq. (29) becomes

$\sum_{n=1}^{\infty} \varepsilon^{n-1}\left[\xi_{b} \sum_{k=1}^{n} k E^{(n-k)}\left[g_{0}\right] \cdot g^{(k)}+2 \xi^{a} n E_{a b}^{(n)}\right]=0$,

where - is for the full contraction. Requesting the analyticity in $\varepsilon$, each coefficient of above equation should be zero. It is useful to write the first few terms explicitly to see the structure,

$$
\begin{aligned}
\xi_{b} E^{(0)}\left[g_{0}\right]{ }^{c d} g_{c d}^{(1)}+2 \xi^{a} E_{a b}^{(1)} & =0, \\
\xi_{b}\left(2 E^{(0)} \cdot g^{(2)}+E^{(1)} \cdot g^{(1)}\right)+4 \xi^{a} E_{a b}^{(2)} & =0, \\
\xi_{b}\left(3 E^{(0)} \cdot g^{(3)}+2 E^{(1)} \cdot g^{(2)}+E^{(2)} \cdot g^{(1)}\right)+6 \xi^{a} E_{a b}^{(3)} & =0,
\end{aligned}
$$

Notice that this is the expansion around the AdS metric $g_{0}$, so that $E^{(0)}\left[g_{0}\right]=0$, which implies $E^{(1)}\left[g_{0}\right]=0$ by the first equation, which in turn implies $E^{(2)}\left[g_{0}\right]=0$ by the second equation. In this way, all $E^{(n)}\left[g_{0}\right]=0$ by the lower ones progressively, proving the whole nonlinear Einstein equation,

$$
\mathbf{E}[g(\varepsilon)]=0,
$$

for all orders in $\varepsilon{ }^{1}$ Therefore, the metric $g(\varepsilon)$ near $g_{0}$ satisfies the full Einstein equation.

\footnotetext{
${ }^{1}$ It is worthwhile to notice that the same conclusion can be derived in more complicated situation where the Eq. (29) is modified to

$$
\hat{E}[g(\varepsilon)] A(\varepsilon)+\hat{C}[g(\varepsilon)] B(\varepsilon)=0,
$$

if the objects $A, B$ have expansion starting from $\varepsilon^{0}$. 
Summarizing, the full Einstein equation holds if and only if the generalized entanglement first law does, thanks to the geometric identity Eq. (27). In other words, the metric $g$ dual to the state $\rho$ compatible with the generalized first law satisfies the nonlinear Einstein equation. Although (24) is derived using the Einstein equation, it is special so that it can imply the Einstein equation itself through the geometric identity. What is the implication of all this? It just means that the relative entropy (RE) expression or the generalized entanglement entropy contains on-shell information. This is clear from the linearized level. There, the first law implies an on-shell condition. The same should be true here. In fact, on the CFT side, the RE can be evaluated only for physical configuration. Therefore, on-shell information is hidden in the entanglement relationship. From the gravity side, the Einstein equation is the criterion for judging whether a given metric configuration is physical. Therefore, it is not surprising that an expression of RE encodes the information of on-shell-ness. After all, the essence of the holography is that the quantum informations of the boundary theory can be encoded by the "on-shell" gravity.

One important remark is that while $\chi$ is a total derivative $\lambda$ on $\tilde{B}$ due to the vanishing of $\xi$ on $\tilde{B}$, it is not so on $B$. Therefore, $\Delta S^{\text {grav }}$ is a total variation but $\Delta E^{\text {grav }}$ is not so in general. This is exactly the same property of $\Delta E, \Delta S$ on the CFT side as we emphasized earlier. However, for an integrable case where $\int_{\Sigma} \xi \cdot \boldsymbol{\omega}=0$, the situation is better, because there exists $K$ and $W_{\xi}$ such that $\xi_{B} \cdot \Theta\left(\frac{d}{d \lambda} g\right)=$ $\frac{d}{d \lambda}\left(\xi_{B} \cdot K\right)$ and $W_{\xi}=\int_{B-\tilde{B}}(\mathbf{Q}-\xi \cdot K)$, respectively [10], so that we can rewrite $(23)$ as $[16,17,23]$

$$
\frac{d}{d \lambda} W_{\xi}=\int_{\Sigma} \omega\left(g ; \frac{d}{d \lambda} g, \delta_{\xi_{B}} g\right)
$$

This can be integrated over $\lambda$ to give

$$
\Delta E_{B}^{\text {grav }}-\Delta S_{B}^{\text {grav }}=\Delta W_{\xi},
$$

where $\Delta$ is a variation from $\rho_{0}$ to $\rho$ whose dual geometries are $g_{0}, g$, respectively. This means that, for an integrable case, the relative entropy is a total variation, and it can be interpreted as the work done on the system to change it from $\rho_{0}$ to $\rho$.

Our method can be easily generalized to the case with inclusion of matter or higher derivatives. For the reference states other than the AdS vacuum, the barrier is the proof of the existence of the Killing vector and its Holland-Wald gauge condition. We leave these matters to future works.

\section{ENTANGLEMENT VECTOR FIELD}

In Ref. [24], the authors tried to reformulate entanglement as the a flux of vector field $\mathbf{v}$. Consider a surface $B^{\prime}$ in $t=0$ slice whose boundary is the same as that of $B$. Our goal is to construct a vector field $V_{E}$ such that

$$
\int_{B^{\prime}} V_{E}^{a} d \sigma_{a}=\int_{\tilde{B}} V_{E}^{a} d \sigma_{a}=S_{B} .
$$

Such a vector field should be divergenceless in the subspace of the $t=0$ slice. Also, it must be a codimension-two form to produce a one-form upon restriction. A natural candidate is $* \mathbf{Q}$ restricted to the constant time slice, and we start from the observation

$$
\int_{\tilde{B}} \mathbf{Q}=S_{B}, \quad d \mathbf{Q}=-\xi \cdot \boldsymbol{\epsilon} L \neq 0,
$$

on shell, where we used Eq. (16) and the fact that $\xi$ is the Killing vector of $g$. Now, we can construct a vector field $V$ by restricting the codimension-two form $\mathbf{Q}$ to the $t=0$ slice. Noticing that among the components of $\xi$, only $\xi^{t}$ is nonzero, we have

$$
16 \pi G_{N} \mathbf{Q}=\nabla^{a} \xi^{b} \epsilon_{a b}=-2 \nabla_{a} \xi^{t} \sqrt{-g_{t t}} \epsilon^{a}:=V_{a} \epsilon^{a} .
$$

In one-form notation, the $V_{a}$ is given by

$$
V=\frac{4 \pi}{R z}\left[\left(\frac{R^{2}-z^{2}-\vec{x}^{2}}{2 z}+z\right) d z+x^{i} d x^{i}\right] .
$$

It is easy to check that $\int_{\tilde{B}} V_{a} \epsilon^{a}=4 \pi \mathrm{Area}[\tilde{B}]$. Therefore, it is tempting to call $V_{a}$ the entanglement vector field. However, for a vector field to be interpreted as a flux, it should be divergenceless so that the flux on the arbitrary surface $B^{\prime}$ is equal to $S_{B}$. Unfortunately, $V$ is not divergence free. In fact, in the $t=0$ slice of $\operatorname{AdS}_{d+1}$,

$$
\nabla_{a} V^{a}=\frac{2 \pi d}{R z}\left(z^{2}+\vec{x}^{2}-R^{2}\right)=(-2 d) n \cdot \xi
$$

where $n$ is the normal vector of the hypersurface $\Sigma$. Furthermore, while we expect that the entanglement vector's flux is highly concentrated at the boundary of the region $B$, the flux of $V$, as one can see in Fig. 1, is almost uniformly distributed over $B$.

Therefore, we look for a balancing vector field $V_{0}$ such that $\nabla_{a}\left(V^{a}-V_{0}^{a}\right)=0$ and flux of $V_{0}$ over $\tilde{B}$ is zero. We take the ansatz $V_{0}=V_{0 r} d r$ and boundary condition

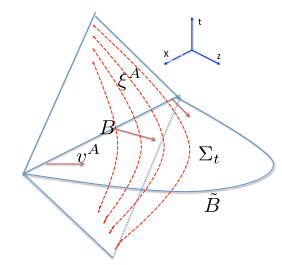

(a)

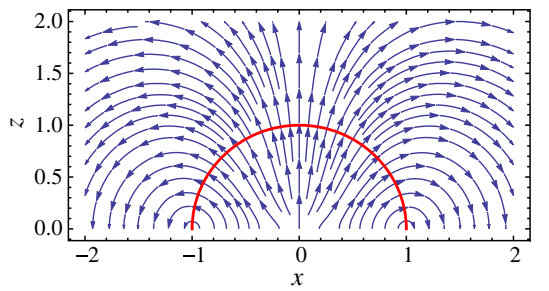

(b)
FIG. 1. (a) Entanglement wedge and flow of vector field $\xi$ and $V$. (b) Flow of vector field $V$ within $\Sigma$. The red circle is the RyuTakayanagi surface. 
$\left.V_{0 r}\right|_{r=R}=0$. One remark is that when we take the divergence of $V$, we should consider $\xi^{t}$ as a scalar once we restrict $\mathbf{Q}$ to the $t=0$ slice. In $\operatorname{AdS}_{d+1}$, it can be given by

$$
V_{0}=\frac{2 \pi d}{R} \frac{(r-R)^{2}}{r^{2} \cos ^{3} \theta} d r
$$

where $r^{2}=z^{2}+\vec{x}^{2}$ and $\cos \theta=z / r$. The final form of the entanglement vector field is give by $V_{E}=V-V_{0}$ whose explicit form in the polar coordinate is

$V_{E}=\frac{2 \pi}{R}\left[\frac{r^{2}+R^{2}}{r^{2} \cos \theta} d r-\frac{\left(R^{2}-r^{2}\right)}{r} \frac{\tan \theta}{\cos \theta} d \theta\right]-V_{0}$

which is a divergence-free vector field whose flux over any $B^{\prime}$ is $S_{B}$ if $B^{\prime}$ is homologous to $B$. One can easily verify that $V_{E}$ satisfies Eq. (37), and for $\mathrm{AdS}_{3}$ the flux of each vector field is

$\int_{B} V_{a} \boldsymbol{\epsilon}^{a}=\frac{c}{9} \frac{R^{2}}{\epsilon^{2}}, \quad \int_{B} V_{0 a} \epsilon^{a}=\frac{c}{9} \frac{R^{2}}{\epsilon^{2}}-\frac{c}{3} \ln \frac{2 R}{\epsilon}$,

where $\epsilon$ is the UV cutoff of $z$ and $c=\frac{3 L}{2 G_{N}}$ with $L$ the AdS radius and $c$ the central charge of the dual $\mathrm{CFT}_{2}$.

Our goal here is to explicitly construct the thread vector of Ref. [24], where the authors suggested replacing the minimal surface by a divergenceless vector. Notice, however, the flux line in Fig. 15 of Ref. [19] similar to our vector field $V$ in Fig. 1 which is not divergenceless. If we impose a zero divergence condition, the resulting vector field $V_{E}$ has the flux lines concentrated at the boundary of the two regions, which reveals an interesting phenomenon: entanglement is done mostly at the boundary of the two entangled regions. As a consequence, the flux of $V_{E}$, as one can see in Fig. 2, looks like sewing the two regions $B$ and $\bar{B}$ along their interface through the holographic direction,

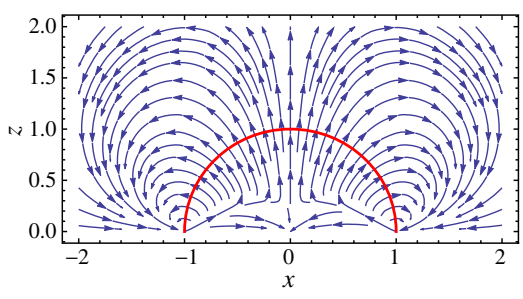

(a)

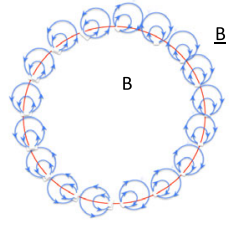

(b)
FIG. 2. (a) Flow of the entanglement vector field $V_{E}$. (b) Cartoon of three-dimensional version of left figure where it is rotated around the $z$ axis.

which is an anticipated feature for the entanglement entropy vector field but was not expected from the general argument of Ref. [24].

\section{DISCUSSION}

We have shown that the generalized entanglement first law implies the full Einstein equation. It would be interesting to study the case in the presence of matter fields or a higher curvature term. We also constructed a vector field $V$ in $\operatorname{AdS}$ space whose flux on the arbitrary surface homologous to $B$ is equal to the entanglement entropy. It would be interesting to utilize the entanglement vector flow to discuss the black hole information problem.

\section{ACKNOWLEDGMENTS}

S. J. S. wants to thank Kimyeong Lee, Sungjae Lee, and Piljin Yi for discussions. This work is supported by the Mid-career Researcher Program through the National Research Foundation of Korea Grant No. NRF2016R1A2B3007687. We appreciate the hospitality of Asia Pacific Center of theoretical Physics (APCTP) during the focus workshop "Geometry and Holography of Quantum Critical Point.”
[1] M. Van Raamsdonk, Building up spacetime with quantum entanglement, Gen. Relativ. Gravit. 42, 2323 (2010); Int. J. Mod. Phys. D 19, 2429 (2010).

[2] J. Maldacena and L. Susskind, Cool horizons for entangled black holes, Fortschr. Phys. 61, 781 (2013).

[3] N. Lashkari, M. B. McDermott, and M. Van Raamsdonk, Gravitational dynamics from entanglement "thermodynamics”, J. High Energy Phys. 04 (2014) 195.

[4] S. Ryu and T. Takayanagi, Aspects of holographic entanglement entropy, J. High Energy Phys. 08 (2006) 045 .
[5] H. Casini, M. Huerta, and R. C. Myers, Towards a derivation of holographic entanglement entropy, J. High Energy Phys. 05 (2011) 036.

[6] T. Faulkner, M. Guica, T. Hartman, R. C. Myers, and M. Van Raamsdonk, Gravitation from entanglement in holographic CFTs, J. High Energy Phys. 03 (2014) 051.

[7] R. M. Wald, Black hole entropy is the Noether charge, Phys. Rev. D 48, R3427 (1993).

[8] V. Iyer and R. M. Wald, Some properties of Noether charge and a proposal for dynamical black hole entropy, Phys. Rev. D 50, 846 (1994). 
[9] V. Iyer and R. M. Wald, A comparison of Noether charge and Euclidean methods for computing the entropy of stationary black holes, Phys. Rev. D 52, 4430 (1995).

[10] R. M. Wald and A. Zoupas, A general definition of "conserved quantities" in general relativity and other theories of gravity, Phys. Rev. D 61, 084027 (2000).

[11] S. Hollands and R. M. Wald, Stability of black holes and black branes, Commun. Math. Phys. 321, 629 (2013).

[12] T. Jacobson, Thermodynamics of Spacetime: The Einstein Equation of State, Phys. Rev. Lett. 75, 1260 (1995).

[13] T. Faulkner, F. M. Haehl, E. Hijano, O. Parrikar, C. Rabideau, and M. Van Raamsdonk, Nonlinear gravity from entanglement in conformal field theories, J. High Energy Phys. 08 (2017) 057.

[14] D. D. Blanco, H. Casini, L. Y. Hung, and R. C. Myers, Relative entropy and holography, J. High Energy Phys. 08 (2013) 060.

[15] J. Lin, M. Marcolli, H. Ooguri, and B. Stoica, Locality of Gravitational Systems from Entanglement of Conformal Field Theories, Phys. Rev. Lett. 114, 221601 (2015).

[16] N. Lashkari, J. Lin, H. Ooguri, B. Stoica, and M. Van Raamsdonk, Gravitational positive energy theorems from information inequalities, Prog. Theor. Exp. Phys. 2016, 12C109 (2016).

[17] N. Lashkari and M. Van Raamsdonk, Canonical energy is quantum Fisher information, J. High Energy Phys. 04 (2016) 153.

[18] B. Swingle and M. Van Raamsdonk, Universality of gravity from entanglement, arXiv:1405.2933.

[19] T. Jacobson, Entanglement Equilibrium and the Einstein Equation, Phys. Rev. Lett. 116, 201101 (2016).

[20] G. Srosi and T. Ugajin, Modular Hamiltonians of excited states, OPE blocks and emergent bulk fields, J. High Energy Phys. 01 (2018) 012.

[21] X. Dong and A. Lewkowycz, Entropy, extremality, Euclidean variations, and the equations of motion, J. High Energy Phys. 01 (2018) 081.

[22] M. Van Raamsdonk, Lectures on gravity and entanglement, arXiv:1609.00026.

[23] D. L. Jafferis, A. Lewkowycz, J. Maldacena, and S. J. Suh, Relative entropy equals bulk relative entropy, J. High Energy Phys. 06 (2016) 004.

[24] M. Freedman and M. Headrick, Bit threads and holographic entanglement, Commun. Math. Phys. 352, 407 (2017). 